\title{
Multiplexed digital mRNA expression analysis profiles system-wide changes in mRNA abundance and responsiveness of UPR-specific gene expression changes during batch culture of recombinant Chinese Hamster Ovary cells \\ DOI:
}

10.1002/biot.201700429

\section{Document Version}

Accepted author manuscript

Link to publication record in Manchester Research Explorer

\section{Citation for published version (APA):}

Dickson, A., \& Maldonado-Agurto, R. (2018). Multiplexed digital mRNA expression analysis profiles system-wide changes in mRNA abundance and responsiveness of UPR-specific gene expression changes during batch culture of recombinant Chinese Hamster Ovary cells. Biotechnology journal. https://doi.org/10.1002/biot.201700429

\section{Published in:}

Biotechnology journal

\section{Citing this paper}

Please note that where the full-text provided on Manchester Research Explorer is the Author Accepted Manuscript or Proof version this may differ from the final Published version. If citing, it is advised that you check and use the publisher's definitive version.

\section{General rights}

Copyright and moral rights for the publications made accessible in the Research Explorer are retained by the authors and/or other copyright owners and it is a condition of accessing publications that users recognise and abide by the legal requirements associated with these rights.

\section{Takedown policy}

If you believe that this document breaches copyright please refer to the University of Manchester's Takedown Procedures [http://man.ac.uk/04Y6Bo] or contact uml.scholarlycommunications@manchester.ac.uk providing relevant details, so we can investigate voukclaim.

\section{OPEEN" ACCESS}




\section{Research Article}

Multiplexed digital mRNA expression analysis profiles system-wide changes in mRNA abundance and responsiveness of UPR-specific gene expression changes during batch culture of recombinant Chinese Hamster Ovary cells ${ }^{\dagger}$

[Rodrigo Maldonado-Agurto ${ }^{1}$

[Alan J. Dickson $]^{2}$

1 [Programa Institucional de Fomento a la Investigación, Desarrollo e Innovación, Univ.

Tecnológica Metropolitana, Ignacio Valdivieso 2409, San Joaquín, P.0. Box, 8940577,

Santiago, Chile.]

${ }^{2}$ [The University of Manchester, Faculty of Life Sciences, Manchester Institute of

Biotechnology, Manchester, United Kingdom]

Correspondence: [Dr. Rodrigo Maldonado-Agurto, Programa Institucional de Fomento a

la Investigación, Desarrollo e Innovación, Univ. Tecnológica Metropolitana, Ignacio

Valdivieso 2409, San Joaquín, P.O. Box, 8940577, Santiago, Chile].

E-mail: [rmaldonado@utem.cl]

Keywords: [Chinese Hamster Ovary (CHO) cells; ER stress; ERAD; NanoString nCounter System; Unfolded Protein Response (UPR)]

${ }^{\dagger}$ This article has been accepted for publication and undergone full peer review but has not been through the copyediting, typesetting, pagination and proofreading process, which may lead to differences between this version and the Version of Record. Please cite this article as doi: [10.1002/biot.201700429].

This article is protected by copyright. All rights reserved

Received: June 14, 2017 / Revised: January 3, 2018 / Accepted: January 4, 2018 


\begin{abstract}
The unfolded protein response (UPR) signalling pathway is viewed as critical for setting the effectiveness of recombinant protein expression in CHO cells. In this study, we used Nanostring nCounter technology to study expression of a group of genes associated with cellular processes linked to UPR activation under ER stress and the changing environment of a batch culture. Time course induction of ER stress, using tunicamycin (TM), showed a group of genes such as Chop, Trb3, Sqstm1, Grp78 and Herpud1 responded rapidly to TM inhibition of N-glycosylation, whilst others such as Atf5, Odz4 and Birc5 exhibited a delayed response. In batch culture, expression of "classical" UPR markers only increased when cells entered decline phase. In addition to providing a detailed analysis of the expression of process-relevant UPR markers during batch culture and in response to imposed chemical stress, we also highlighted six genes (Herpud1, Odz4, Sqstm1, Trb3, Syvn1 and Birc5) associated with the perception of ER stress responses in recombinant CHO cells. Herpud1 (involved in ER-associated degradation) exhibited a rapid (primary) response to stress and its relationship (and that of the other five genes) to the overall cellular UPR may identify novel targets to modulate recombinant protein production in $\mathrm{CHO}$ cells.
\end{abstract}

Abbreviations: [ATF6(N), ATF6 nuclear, UPR, unfolded protein response; TM, tunicamycin; ERAD, ER-associated protein degradation; EPO, Erythropoietin; RP, recombinant protein; PERK, PRKR-like ER kinase; PtdCho, phosphatidylcholine] 


\section{Introduction}

The accumulation of abnormally folded proteins in the ER lumen, a cellular condition referred to as ER stress, activates the UPR to restore proteostasis [1]. The UPR integrates information about the intensity and duration of the stimuli, engaging adaptive programs to reduce the unfolded protein load. This adaptive response involves the upregulation of several ER chaperones and foldases, inhibition of general protein translation and increased phospholipid synthesis with subsequent ER membrane expansion. Additionally, degradation of abnormally folded proteins through the ER-associated protein degradation (ERAD) pathway and autophagy is increased. Failure to overcome ER stress, however, engages the core apoptosis machinery [2]. Cells in batch culture are exposed to a combination of stimuli that can activate the UPR, especially during late stages of cell culture. Whilst recombinant protein (RP) expression may be an important stimulus, conditions such as nutrient depletion (i.e. glucose) [3], osmotic stress [4] and oxidative stress [5] have also been shown to trigger UPR. Several components of the UPR signalling pathway have been engineered in mammalian cells with the aim of increasing RP production. Specifically, these include a number of chaperones such as BIP [6], calnexin and calreticulin [7], members of the PDI family [8] and components of the UPR signalling pathway such as CHOP [9], ATF4 [10], [11], GADD34 [12], XBP-1. Nevertheless, the results have shown to be process-specific, depending on the protein expressed and type of expression (stable, transient). Steps in the UPR-related pathway(s) have been promoted as targets for cell-engineering to improve recombinant protein expression, but the complexity of know interactions and lack of predictability of the engineered phenotypes reflects upon our limited understanding of the key events of UPR (and the interacting system). In addition to the complexity and limited understanding of the signalling pathways involved in UPR regulation, there are specific technical issues associated with studying these phenomena in Chinese Hamster Ovary (CHO) cells. The limited availability of antibodies against many potentially important gene 
targets (in order to profile changes and timings to define molecular events) in general is further compounded by the failure of many commercially-available antibodies to crossreact with proteins extracted from $\mathrm{CHO}$ cells [4]. Hence the idea of screening for changes at protein level using western blotting is restricted due to the lack of panels of relevant antibodies (an aspect we experienced). Definition of targets at mRNA level is a pre-requisite to detailed studies of the roles of specific genes at protein level. Such information will focus on development of antibody reagents to support molecular analyses (including knock-in/out approaches) to develop a systems-level understanding of the regulatory phenotype associated with $\mathrm{CHO}$ cell capability as a protein expression platform. This work aimed to define the profile of change of specific targets whilst showing the potential for multiplexing RNA assessment of gene expression. The multiplexed RNA assessment enables a focus on genes that would repay optimisation of western blotting processes, either by optimisation of the use of commercially available antibody reagents or by production of specific antibodies raised specifically against immunogenic epitopes on $\mathrm{CHO}$ variants of the defined targets. The goal of this study was to profile of simultaneous changes of a set of UPR-related mRNA species during batch culture and ER stress. In order to do this, we used the Nanostring nCounter system which has been successfully applied to profile mRNA expression in CHO cells [13]. Unlike standard qRT-PCR, the Nanostring nCounter allows the quantitative measurement of expression and relative abundance of up to 800 transcripts simultaneously without staged conversion of mRNA to cDNA or the amplification of the resulting cDNA by PCR [14]. Cell-to-cell variation have been shown for a variety of gross CHO cell characteristics including cell growth[15], protein glycosylation[16], response to culture environment[17], metabolism[18] and UPR activation (timing and extent)[4]. In this context, -omics studies of clonal derivatives have found that clonal variation may be more important than recombinant protein expression to the differences observed in metabolomics or transcriptomics studies [18], [19]. For this reason in the presented work 
we did not compared the mRNA expression patterns between different clones. Instead, in this study we followed the changes in mRNA abundance expression for a selected group of 27 genes associated with different ER stress-related processes including UPR signalling, ERassociated protein degradation (ERAD) and autophagy, in a model cell line during 10 days of batch culture and at different times after ER stress induction, by the N-glycosylation inhibitor, tunicamycin (TM). Our results show that the abundance of measured UPR-related mRNAs varies by up to 4 orders of magnitude and that their responsiveness to TM-induced ER stress also varies greatly. In this respect, genes associated with different cellular processes such as the PERK signalling pathway, ERAD and autophagy were shown to be very responsive to TM-induced ER stress in CHO cells. These results highlight the widespread gene expression alteration caused by ER stress across multiple cellular events, the differential extent of the responses to distinct formats of stress and highlight the temporal relationship between the regulation of mRNA encoding distinct arms of the UPR, ERAD and autophagy pathways.

\section{Materials and Methods}

\subsection{Cell lines and Cell Culture}

Recombinant CHO-DG44 cell lines expressing erythropoietin (CHO-EPO) were transfected and methotrexate (MTX) amplified as described previously [20]. Adherent recombinant CHO-DG44 cell lines were initially adapted to serum-free suspension culture for this study. In routine culture (sub-cultured every 3 days) and batch experiments CHOEPO cells were seeded at $2 \times 10^{5}$ cells $/ \mathrm{ml}$ in $30 \mathrm{ml}$ of medium in $125-\mathrm{mL}$ shake flasks (Corning), and cultured with shaking (130 rpm) in a humidified incubator at $37^{\circ} \mathrm{C}$ and $5 \%$ $\mathrm{CO}_{2}$. The medium was $\mathrm{CD}$ OptiCHO ${ }^{\mathrm{TM}}$ medium (Invitrogen,) supplemented with $1 \mathrm{x}$ Glutamax (Gibco) and $250 \mathrm{nM}$ MTX. For batch culture experiments, CHO-EPO cells (in triplicate) were sampled daily to determine viable cell density and recombinant erythropoietin (EPO) (from medium samples). mRNA was also isolated on specific days of culture (as described in 
Section 2.3). For TM treatment, CHO-EPO cell lines were seeded at $2 \times 10^{5}$ cells $/ \mathrm{ml}$ and cultured for 3 days, then TM (Sigma-Aldrich) was added at a final concentration of $6 \mu \mathrm{g} / \mathrm{ml}$ and RNA was extracted at 4, 8, and $24 \mathrm{~h}$ after treatment and used for expression analysis. Controls (without TM) were harvested in parallel. Cell density and viability was assessed by trypan blue exclusion using a Neubauer counting chamber.

\subsection{ELISA}

Secreted recombinant human erythropoietin (rhEPO) was quantified using a sandwich ELISA. 96-well immunoplates (Nunc) were coated with mouse anti-hEPO antibody (500 $\mu \mathrm{g} / \mathrm{mL}$, MAB287; R\&D Systems) and incubated for $16 \mathrm{~h}$ at $4^{\circ} \mathrm{C}$. The following day, the coated plates were washed with washing buffer $(0.05 \%[\mathrm{v} / \mathrm{v}]$ Tween-20, 0.05\% [v/v] phenol red solution in sterile PBS) and blocked with blocking solution (25\% [w/v] BSA, 0.4\% [w/v] sodium azide in sterile PBS) for one hour at room temperature. After blocking, plates were washed with washing buffer. The standard and medium samples $(1: 10000-1: 40000$ dilution) were diluted in dilution buffer (1\% [w/v] BSA in sterile PBS) and applied to plates in triplicate. rhEPO produced in CHO cells (Calbiochem, product number 329871) was used as a standard. For detection, a polyclonal IgG anti-hEPO (R\&D Systems, MAB 286) was applied, followed by the addition of a goat anti-rabbit IgG horseradish peroxidase conjugate antibody (Sigma-Aldrich). The reaction was developed using TMB substrate solution (3,3',5,5' Tetramethyl Benzidine Chromogen, Sigma-Aldrich) prepared according to the manufacturer's instructions. After washing six times, TMB substrate solution was added and wells were incubated at room temperature for 20-30 minutes in dark. The reaction was stopped by addition of $0.2 \mathrm{M} \mathrm{H}_{2} \mathrm{SO}_{4}$ and the absorbance was measured at $450 \mathrm{~nm}$ using a BioTek Powerwave 340 plate reader (Biotek). Sample concentration was calculated from the standard. 


\subsection{RNA isolation and characterisation}

Total RNA was isolated from $5 \times 10^{6}$ cells using TRIzolTM(Invitrogen) as per the manufacturer's instructions. A NanoDrop® ND-1000 UV-Vis (Thermo Scientific) was used to quantify RNA, and $10 \mu \mathrm{g}$ samples of RNA were treated with DNase I (RNase-free) (New England Biolabs), according to the manufacturer's instructions. Further purification of the DNase I treated RNA was performed using RNeasy Mini Kits (Qiagen) according to manufacturer's instructions. A final RNA quantity and quality assessment was performed by using the TapeStation 2200 (Agilent Technologies).

\section{4 nCounter analysis}

NanoString nCounter CodeSets for 31 different genes were designed and synthesized by NanoString Technologies. A panel of 27 UPR-associated genes and 4 housekeeping genes were used for nCounter analysis (NanoString Technologies). By reference to the literature, we selected genes associated with specific processes within the overall heading of UPRrelated genes and grouped them as follows: PERK signalling pathway (9 genes), ER chaperones (4 genes), phosphatidylcholine synthesis pathway (PtdCho) enzymes (6 genes), ER-associated degradation (5 genes), Autophagy-related genes (3 genes) along with 4 housekeeping genes (Table 1). Expression analysis was performed by the Genomic Technologies Core Facility, (Faculty of Life Science, The University of Manchester). NanoString nCounter gene expression analysis was performed using two specific probes (capture and reporter) for each gene of interest. In brief, hybridization of $100 \mathrm{ng}$ of RNA (per sample) with customized Reporter CodeSet and Capture ProbeSet was performed in a NanoString Prep Station (NanoString Technologies) and the mRNA molecules counted with the NanoString nCounter (NanoString Technologies). The nSolver ${ }^{\mathrm{TM}}$ Analysis Software 3.0 (NanoString Technologies) was used to perform data handling, including automated background subtraction, spike-in-control normalization and reference gene normalization. Furthermore, datasets from duplicates were grouped and fold change estimates were 
calculated by building ratios, setting as 1 the mRNA expression on day 1 (for batch culture experiments) and the controls without TM (for TM-induction experiments).

\subsection{Statistical analysis}

Data presented are shown either as fold-changes or as percentages with mean \pm S.D. (standard deviation) or S.E.M. (standard error of mean), as indicated in the Figure legends. Statistical analyses were performed using GraphPad Prism version 6.00 for Mac (GraphPad Software, San Diego California USA, www.graphpad.com). Unpaired Student's t-test was used for statistical analysis.

\section{Results}

\subsection{Characterisation of recombinant CHO-EPO cells and relative abundance of UPR-} related mRNA during exponential phase of batch culture

Cell growth and volumetric EPO productivity was measured throughout batch culture (Figure 1A). Specific productivity of this cell line (28 pg EPO/cell/day) is comparable to that determined for other EPO-producing CHO cell lines [21]-[23]. This cell line provides a model in which the exponential phase lasted until day 6, with a subsequent stationary phase of growth that continued until day 8, after which cells entered a decline phase (Figure 1B). The panel of 27 UPR-associated genes and 4 housekeeping genes (Table 1) were used for NanoString nCounter analysis. Figure 1C shows the relative abundance of mRNAs measured by NanoString nCounter during the exponential phase of growth (day 3). mRNA abundance varied from gene to gene and most mRNAs were generally low compared to the housekeeping genes Actb and Gapdh. Besides the housekeeping genes, the most abundant mRNAs identified were those of the ER chaperone category (Grp94, Grp78 and Pdi) and the transcription factor Atf4. The range of mRNA molecules detected is in agreement with previously reported data where the abundance of mRNAs showed counts from 2 to $\sim 60,000$ in recombinant $\mathrm{CHO}$ cells [13]. 


\subsection{Use of NanoString nCounter analysis to profile responsiveness of UPR-related}

genes to tunicamycin-induced ER stress

The treatment of recombinant CHO-EPO cells with TM during the exponential phase of the culture (when nutrients are not limiting) was used to induce the activation of the UPR [24] and determine which of the panel of genes responded to chemically-induced ER-stress. In the presence of tunicamycin, cell growth was slowed by less than $10 \%$ and cell viability was similar between control and tunicamycin-treated cells over the period of sampling for RNA assessment. The NanoString nCounter analysis for each time point before and after induction was performed on biological duplicates (Figure 2). After normalization of the replicates, genes that increased or decreased by 1.5 fold or more (with a t-test score of $\leq 0.05$ ) were categorised to be differentially expressed. Fourteen genes significantly changed their expression in response to TM treatment. Among these, thirteen genes (Chop, Trb3, Odz4, Gadd34, Grp78, Grp94, Xbp1, Ulk1, Wipi1, Sqstm1, Sels, Syvn and Herpud1) were up-regulated and one (Birc5) was down-regulated. The genes associated with the synthesis of phosphatidylcholine (PtdCho) showed limited change in expression (less than 2 fold increase) following chemical ER stress imposition. We observed two different patterns of mRNA change upon TM addition, with mRNA for genes such as Chop and Trb3 rapidly increasing in abundance between 0.5 and $4 \mathrm{~h}$ of treatment, with maintenance of expression for up to $24 \mathrm{~h}$ after TM treatment. On the other hand, mRNAs encoding for Wipi1 and Ulk1 gradually increased in amount, only peak abundance only after $24 \mathrm{~h}$ of induction (the last time of measurement).

\subsection{Use of NanoString nCounter analysis to profile UPR-related genes during batch}

\section{culture}

The expression of the same panel of mRNAs was measured throughout batch culture to provide a molecular characterisation of extent to which chemical and environmental stresses provoke common or distinct readouts of cellular status, an approach that provides 
the potential to define template targets to study $\mathrm{CHO}$ cell well-being in culture (Figure 3). RNA samples were collected on days 1, 3, 6 (exponential), 7, 8 (stationary), 9 and 10 (decline) of batch culture. NanoString nCounter analysis was performed with biological duplicate samples. Figure 3 shows the mRNA amount during batch culture, relative to each specific mRNA amount on day 1. Out of the twenty-seven mRNAs studied, Chop, Trb3, Odz4, Grp78, Grp94, Sqstm1, Sels and Herpud1 increased as the culture progressed and Birc5 mRNA decreased, whilst Gadd34, Xbp1, Ulk1, Wipi1 and Syvn1 remained constant throughout the culture. The genes associated to the PtdCho synthesis pathway remained constant throughout batch culture.

\section{Discussion}

\subsection{Chaperone mRNAs are highly abundant in the recombinant CHO cell line}

To define differences between stress perception associated with physiological batch culture stress and chemically-induced UPR stress, we assessed the gene (mRNA) expression prolife of twenty-seven UPR-related mRNAs during batch culture and after incubation with the Nglycosylation inhibitor, tunicamycin. The genes were selected due to their association with a range of cellular events that occur as a consequence of the accumulation of misfolded proteins in the ER. The simultaneous quantification of several UPR-related mRNAs showed that the abundance of the studied mRNAs in unstressed cells (during exponential phase of growth) varied by orders of magnitude, with the top 3 most abundant mRNAs encoding Grp78/Bip (that belong to the protein-folding category) and Atf4 (a member of the PERK signalling pathway). In addition to the chaperones Grp78/Bip and Grp94, the protein-folding category included the fourth most abundant mRNA, that encodes a protein disulphide isomerase (Pdi) which catalyses disulphide bond formation. It is highly informative that these findings, at mRNA level, are in agreement with proteomic data, where it has been shown that GRP78/BiP, GRP94 and PDI are the most abundant ER proteins in different cell types, including CHO cells [25]. On the other hand, Atf4, the third more abundant mRNA is 
a common player for multiple cellular stresses including amino acid metabolism regulation, cellular redox state, and anti-stress responses [26].

\subsection{Selective responsiveness of UPR-related mRNA panel to TM-induced ER stress}

TM-induced ER stress significantly changed the expression of fourteen genes, five belonging to the PERK signalling pathway category (Chop, Trb3, Odz4, Gadd34 and Birc5), three to the protein folding (Grp78, Grp94 and Xbp1), three to the autophagy-related genes (Ulk1, Wipi1 and Sqstm1) and three to the ERAD (Sels, Syvn and Herpud1). The activation of the UPR arms associated with the transcription factors spliced X-box binding protein 1 (XBP1s) and activating transcription factor $6 \alpha(\mathrm{ATF} 6 \alpha)$ have been linked to ER biogenesis [27]-[30], indicating that the UPR may have the potential to enhance ER capacity by expanding its size[27]-[29], [31]. Therefore, it was reasoned that if the UPR is activated by TM, XBP1s and ATF6 $\alpha$ activation would also alter the expression of mRNA of enzymes of the PtdCho pathway. Nevertheless, no changes to the quantity of the mRNAs were observed in the PtdCho pathway genes after the addition of tunicamycin, which is in agreement with the findings from previous studies that show that the major control of enzymatic activity of the PtdCho synthesis pathway occurs post-translationally [32]. Several previous reports have observed transcriptional activation of UPR target genes and proteins during the culture of cell lines expressing a RP and in parental CHO cell lines [3], [4], [33], [34]. In this study, mRNAs associated with the activation of the PERK arm of the UPR (i.e. protein folding, autophagy and ERAD) were observed to increase in abundance as batch culture progressed, with these mRNAs showing a commonality of response to that observed with tunicamycin addition. Chop, Trb3, Grp78, Sqstm1 and Herpud1 presented maximum responsiveness to TM after 4 hours of treatment, making them highly interesting early targets to monitor ER stress. The idea of an adaptive and a chronic stress response differentiated by pro-survival and pro-apoptotic signalling suggested the expression of mRNAs associated with different cellular processes would provide a view of the cellular state[35], [36]. Nevertheless, in the 
timeframe of cell culture studied, only the up-regulation that represents the adaptive response of the UPR was observed. Even though the mechanism controlling the decision between adaptive and chronic (cell death) responses is not fully understood, it has been established that, depending on the extent of ER stress (time and amplitude), a differential activation occurs for specific components of the UPR pathway. For example, it is known that downstream targets of PERK cause translation recovery [37], ROS production [38] and the expression of pro-apoptotic genes (i.e. BIM, PUMA) [39]. It has been shown that the transition from an adaptive UPR to ER stress-induced apoptosis during chronic ER stress occurs when IRE1 $\alpha$ and ATF6 activities are attenuated, whilst PERK signalling is maintained [36], [40]. Thus, the fact that only the adaptive response was observed at mRNA level was detected (after TM treatment and during batch culture) may indicate that the stress was not strong or prolonged enough to cause attenuation of the certain arms of the UPR with the consequent induction of cell death[5]. The use of more severe (or prolonged) stress conditions (that disrupt ER function through different mechanisms) could be used to evoke the activation of pro-apoptotic cascades. ER stress, producing distinct temporal kinetics and activation of further UPR branches [41], [42]. Likewise, the overexpression of the active version of the ER stress sensors [40] or the associated transcription factors (XBP1s, ATF4 and ATF6 (N)) may be used to selectively activate the UPR [38], [43]. Moreover, expression of recombinant proteins such as Factor VIII has been reported to induced ER stress, UPR activation and apoptosis in CHO cells [5]. Additionally, the use of inducible RP expression systems (that induce ER stress) or removal of the stressor could serve as a model of ER stress recovery and allow greater insight into the combinatorial regulatory events. 


\subsection{The importance of protein degradation pathways for recombinant protein production}

In mammalian cells the majority of misfolded proteins in the ER are eliminated through either the ubiquitin/proteasome pathway known as ERAD (I) or the autophagic/lysosomal pathway known as ERAD (II)[44]. Recent publications have shown the activation of autophagy in CHO cells during nutrient deprivation [45], sodium butyrate addition [46] and hyperosmotic stress [47]. Here, it has been shown that the induction of the UPR activation by addition of TM increased the abundance of mRNA encoding for genes associated with autophagy (Ulk1, Wipi1, Sqstm1) and that batch culture modestly increased their expression during the decline phase. Although both ERAD pathways are active in CHO cells [48], only autophagy has been exploited towards improvement of recombinant protein production [49]. In our model cell line, the time course of activation of Chop and Trb3 (PERK pathway) mRNA expression was similar to that was observed for Syvn1 and Herpud1 (ERAD pathway), with greatest mRNA observed after $4 \mathrm{~h}$ treatment, a response maintained up to $24 \mathrm{~h}$ of induction. On the other hand, genes linked to autophagy such as Ulk1, Wipi1 and Sqstm1 increased their abundance gradually between $4 \mathrm{~h}$ and $24 \mathrm{~h}$ after TM addition. Other genes such as $0 d z 4, A t f 5$ and Birc5 only presented differences in expression 24h after treatment with TM. These data exhibit the power of Nanostring nCounter technology to provide simultaneous profiling of temporal interconnected cellular response pathways.

\subsection{Transcriptional regulation of UPR-related genes}

Phosphorylation plays a major role in the regulation of the UPR, being responsible for the activation of the stress sensors located in the ER membrane. These are responsible for sensing the state of ER lumen and transmit the response to the nucleus to regulate transcription of genes that can help to restore ER homeostasis. Whilst protein phosphorylation analysis presents one aspect of control, the amount of specific mRNAs represent an further integrated assessment of the engagement of all regulatory events. The 
three transcription factors (XBP1s, ATF6(N), and ATF4) generated from activation of three UPR sensors pathways localize to the nucleus where they bind the promoters of UPRspecific genes. These ER stress responsive cis-acting elements are the ER stress response element (ERSE, CCAAT-N9-CCACG) [50], ERSE-II [51] (which is very similar to ERSE except that it is separated by a single nucleotide spacer), UPR response element (UPRE, TGACGTGG/A) and C/EBP-ATF Response Element (CARE, TTGCATCA) also called Amino Acid Response Elements (AARE) [52](Figure 4). The promoter region of UPR-responsive genes present one or more ER stress-responsive cis-acting elements, which determines their response to one or more UPR pathways. Regarding the regulation of the genes from this study, it is known that all three branches of the UPR can regulate the transcription of Chop, which encodes a transcription factor that controls the expression of several target genes including $O d z 4$ and Trb3. The Trb3 promoter present a functional AARE to which both ATF4 and CHOP can bind and controls its transcription depending on the stimuli [53]. Odz4 was first described as a downstream target of $\mathrm{CHOP}$, and recently has gained attention due to a miRNA (miR-708) encoded within an intron of this gene [54]. It has been shown that miR-708 may induce apoptosis through the downregulation of the mRNA encoding for the apoptosis inhibitor protein BIRC5 (also know as Survivin) [55]. The promoter of the Sqstm1 gene is regulated by several factors [56]. However, in the context of amino acid starvation and ER stress, it has been shown that ATF4 and CHOP bind to the AARE to activate Sqstm1 transcription [57]. Hrd1 expression is regulated by ATF6 and XBP1s, both of which can interact with different UPRE and ERSE elements present in the promoter [58]. The ER stress sensors PERK, ATF6, and IRE1 are often considered to act in parallel, and each branch of the UPR may exert selective control on the expression of a set of target genes associated with specific cellular processes such as protein folding, ERAD and apoptosis. Nevertheless, emerging evidence shows cross-regulation between the UPR branches. As example of this cross-talk, the Herpud-1 promoter contains the ER stress-responsive cis- 
acting elements C/EBP-ATF, ERSE-II, and ERSE-I. ATF4 can bind to C/EBP-ATF and although this binding is not essential for Herpud1 induction during ER stress, the binding is needed for maximal induction[59].XBP1s and ATF6(N) can both regulate the expression of Herpud1 by binding to the ERSE and ERSEII sequences in the promoter, in a NF-Y dependent and/or independent manner (Figure 4). Therefore, these three signalling pathways converge in the Herpud1 promoter and are needed for its maximal induction, suggesting a previously-undetermined but important role for Herpud1 in the control of the ER homeostasis.

\subsection{The overall significance of these data}

Despite the changes in mRNA expression observed in this study, antibodies are needed in order to be able to associate the expression of certain mRNA with the onset of a cellular process (i.e phosphorylation). Commercial antibodies are available against some UPR target proteins. However, many of them have been raised against epitopes from human or rodent amino acid sequences. Consequently, some antibodies that may be thought of as relevant to analysis of $\mathrm{CHO}$ proteins turn out to produce no signals or exhibit cross-reaction with non-specific proteins. Our approach is one of providing the spotlight to define those targets upon which to focus subsequent effort. We would like to reiterate that this is a significant undertaking and does not represent an off-the-shelf answer to studying the target proteins.

In this study, the amounts of mRNAs that rapidly respond to the status of the ER were measured. These mRNAs profile a set of UPR-responding genes linked to different cellular events that occur upon the activation of UPR. We are aware that the precise conditions of batch culture will present an environmental descriptor of stress (e.g. lactate or ammonia accumulation, $\mathrm{pH}$ ) but these have not been measured in the current study. With the knowledge of specific mRNA responses, it is easy to envisage as series of experiments that 
would screen specific environmental stresses to a profile of specific members of this subset of mRNAs.

The sensitivity to ER and environmental stress by different $\mathrm{CHO}$ cell clones, expression of different RPs or different culture conditions may all be assessed by multiplexed profiling of expression of a small sub-set of mRNAs. Whilst the NanoString nCounter has the capacity to quantify a large subset of mRNAs belonging to varied and linked pathways, we have used the current studies to define mRNAs that are most likely to provide an informative fingerprint of cellular state. The smaller subset offers the potential for exploitation to interrogate a range of cellular challenges such as sodium butyrate treatment [13], hyperosmotic conditions [60] or elevated ammonium concentrations [61]. Despite the profiling observed in this study, further work will be needed to refine the association between mRNA expression patterns and cellular phenotypes (and to provide deeper understanding of molecular descriptions of cellular phenotype). For example, Tsuyuki et al. found that Wipi1 mRNA expression correlated with autophagosome accumulation in human fibroblasts (WI-38 and TIG-1), human cancer cells (U-2 OS, Saos-2, and MCF7), and rodent fibroblasts (Rat-1) treated with different autophagy inducers. This finding suggests that detection of Wipi1 mRNA could be used to monitor autophagosome formation in a wide range of cell types [62]. Genome editing offers the potential to use endogenous promoters of responsive genes such as Grp78 to monitor the transcriptional regulation of endogenous gene expression in its native chromatin landscape, during cell line development, for example [63]. These approaches may be applied to monitor the different cellular processes associated with the UPR pathway, in order to quantify and dissect how the culture environment affect $\mathrm{CHO}$ cells. Further investigation will focus on understanding the function of the proteins encoded by these mRNAs during cell culture. Exploitation of genes and/or promoters that present a rapid and high response to conditions that alter the ER function may be used to improve recombinant protein production in CHO cells, through cell 
engineering (i.e ERAD engineering) or the application of this knowledge towards the design of in vivo stress sensors (i.e. autophagy sensor) to monitor the cellular status during production process development.

\section{Acknowledgement}

R.M. gratefully acknowledges the financial support from Becas Chile scholarship (Grant 72101007) granted by CONICYT-Chilean government. We would also like to thank Dr Zeynep Betts for providing the recombinant CHO cell lines and the University of Manchester Genomic Technologies Core Facility for the Nanostring nCounter analysis.

\section{Conflict of interest}

The authors declare no financial or commercial conflict of interest. 


\section{References}

[1] C. Hetz, E. Chevet, and S. A. Oakes, Nat. Cell Biol., 2015, 17, 829.

[2] I. Tabas and D. Ron, Nat. Cell Biol., 2011, 13, 184.

[3] T. C. Murphy, N. R. Woods, and A. J. Dickson, Biotechnol. Bioeng., 2001, 75, 621.

[4] Z. Du, D. Treiber, R. E. McCoy, A. K. Miller, M. Han, F. He, S. Domnitz, C. Heath, and P. Reddy, Biotechnol. Bioeng., 2013, 110, 2184.

[5] J. D. Malhotra, H. Miao, K. Zhang, A. Wolfson, S. Pennathur, S. W. Pipe, and R. J. Kaufman, Proc. Natl. Acad. Sci. U. S. A., 2008, 105, 18525.

[6] N. Borth, D. Mattanovich, R. Kunert, and H. Katinger, Biotechnol. Prog., 2008, 21, 106.

[7] J. Y. Chung, S. W. Lim, Y. J. Hong, S. O. Hwang, and G. M. Lee, Biotechnol. Bioeng., 2004, 85, 539.

[8] C. Mohan, Y.-G. Kim, J. Koo, and G. M. Lee, Biotechnol. J., 2008, 3, 624.

[9] D. Nishimiya, T. Mano, K. Miyadai, H. Yoshida, and T. Takahashi, Appl. Microbiol. Biotechnol., 2013, 97, 2531.

[10] A. M. Haredy, A. Nishizawa, K. Honda, T. Ohya, H. Ohtake, and T. Omasa, Cytotechnology, 2013, 65, 993.

[11] T. Ohya, T. Hayashi, E. Kiyama, H. Nishii, H. Miki, K. Kobayashi, K. Honda, T. Omasa, and H. Ohtake, Biotechnol. Bioeng., 2008, 100, 317.

[12] T. Omasa, T. Takami, T. Ohya, E. Kiyama, T. Hayashi, H. Nishii, H. Miki, K. Kobayashi, K. Honda, and H. Ohtake, J. Biosci. Bioeng., 2008, 106, 568.

[13] S. M. Lee, Y. G. Kim, E. G. Lee, and G. M. Lee, J. Biotechnol., 2014, 171, 56.

[14] G. K. Geiss, R. E. Bumgarner, B. Birditt, T. Dahl, N. Dowidar, D. L. Dunaway, H. P. Fell, S. Ferree, R. D. George, T. Grogan, J. J. James, M. Maysuria, J. D. Mitton, P. Oliveri, J. L. Osborn, T. Peng, A. L. Ratcliffe, P. J. Webster, E. H. Davidson, and L. Hood, Nat. Biotechnol., 2008, 26, 317. 
[15] L. M. Barnes, N. Moy, and A. J. Dickson, Biotechnol. Bioeng., 2006, 94, 530.

[16] S. L. Davies, C. S. Lovelady, R. K. Grainger, A. J. Racher, R. J. Young, and D. C. James, Biotechnol. Bioeng., 2013, 110, 260.

[17] S. K. Yoon, S. O. Hwang, and M. L. Gyun, Biotechnol. Prog., 2004, 20, 1683.

[18] A. Ghorbaniaghdam, J. Chen, O. Henry, and M. Jolicoeur, PLoS One, 2014, 9.

[19] M. K. Oh, D. R. Scoles, C. Haipek, A. D. Strand, D. H. Gutmann, J. M. Olson, and S. M. Pulst, J. Cell. Biochem., 2003, 90, 1068.

[20] Z. Betts and A. J. Dickson, Mol. Biotechnol., 2015, 57, 846.

[21] W. S. Ahn, J.-J. Jeon, Y.-R. Jeong, S. J. Lee, and S. K. Yoon, Biotechnol. Bioeng., 2008, $101,1234$.

[22] S. K. Yoon, J. Y. Song, and G. M. Lee, Biotechnol. Bioeng., 2003, 82, 289.

[23] S. K. Yoon, J. K. Hong, and G. M. Lee, Biotechnol. Prog., 2004, 20, 1293.

[24] H. P. Harding, Y. Zhang, and D. Ron, Nature, 1999, 397, 271.

[25] T. Gidalevitz, F. Stevens, and Y. Argon, "Orchestration of secretory protein folding by ER chaperones," Biochimica et Biophysica Acta - Molecular Cell Research, 1833. 2410, 2013.

[26] J. Ye and C. Koumenis, Curr. Mol. Med., 2009, 9, 411.

[27] A. L. Shaffer, M. Shapiro-Shelef, N. N. Iwakoshi, A. H. Lee, S. B. Qian, H. Zhao, X. Yu, L. Yang, B. K. Tan, A. Rosenwald, E. M. Hurt, E. Petroulakis, N. Sonenberg, J. W. Yewdell, K. Calame, L. H. Glimcher, and L. M. Staudt, Immunity, 2004, 21, 81.

[28] R. Sriburi, S. Jackowski, K. Mori, and J. W. Brewer, J. Cell Biol., 2004, 167, 35.

[29] R. Sriburi, H. Bommiasamy, G. L. Buldak, G. R. Robbins, M. Frank, S. Jackowski, and J. W. Brewer, J. Biol. Chem., 2007, 282, 7024.

[30] H. Bommiasamy, S. H. Back, P. Fagone, K. Lee, S. Meshinchi, E. Vink, R. Sriburi, M. Frank, S. Jackowski, R. J. Kaufman, and J. W. Brewer, J. Cell Sci., 2009, 122, 1626.

[31] M. Tigges and M. Fussenegger, Metab. Eng., 2006, 8, 264. 
[32] M. V Barone, A. Crozat, A. Tabaee, L. Philipson, and D. Ron, Genes Dev. , 1994, 8, 453.

[33] M. F. Underhill, J. R. Birch, C. M. Smales, and L. H. Naylor, Biotechnol. Bioeng., 2005, $89,805$.

[34] K. Prashad and S. Mehra, Cytotechnology, 2015, 67, 237.

[35] U. Woehlbier and C. Hetz, "Modulating stress responses by the UPRosome: A matter of life and death," Trends in Biochemical Sciences, 36. 329, 2011.

[36] J. H. Lin, H. Li, D. Yasumura, H. R. Cohen, C. Zhang, B. Panning, K. M. Shokat, M. M. Lavail, and P. Walter, Science, 2007, 318, 944.

[37] E. Kojima, A. Takeuchi, M. Haneda, F. Yagi, T. Hasegawa, K. Yamaki, K. Takeda, S. Akira, K. Shimokata, and K. Isobe, FASEB J., 2003, 17, 1573.

[38] J. Han, S. H. Back, J. Hur, Y.-H. Lin, R. Gildersleeve, J. Shan, C. L. Yuan, D. Krokowski, S. Wang, M. Hatzoglou, M. S. Kilberg, M. A. Sartor, and R. J. Kaufman, Nat. Cell Biol., 2013, 15, 481.

[39] K. D. McCullough, J. L. Martindale, L.-O. Klotz, T.-Y. Aw, and N. J. Holbrook, Mol. Cell. Biol. , 2001, 21, 1249.

[40] J. H. Lin, H. Li, Y. Zhang, D. Ron, and P. Walter, PLoS One, 2009, 4.

[41] and M. N. Jenny B. DuRose, Arvin B. Tam, Mol. Biol. Cell, 2007, 18, 986.

[42] S. S. Shinjo, Y. M. Izotani, E. T. Ashiro, and M. I. Moto, Biosci. Biotechnol. Biochem., 2013, 77, 729 .

[43] M. D. Shoulders, L. M. Ryno, J. C. Genereux, J. J. Moresco, P. G. Tu, C. Wu, J. R. Yates, A. I. Su, J. W. Kelly, and R. L. Wiseman, Cell Rep., 2013, 3, 1279.

[44] E. Fujita, Y. Kouroku, A. Isoai, H. Kumagai, A. Misutani, C. Matsuda, Y. K. Hayashi, and T. Momoi, Hum. Mol. Genet., 2007, 16, 618.

[45] S. O. Hwang and G. M. Lee, Biotechnol. Bioeng., 2008, 99, 678.

[46] J. Seong Lee and G. M. Lee, Biotechnol. Prog., 2012, 28, 349.

[47] Y. K. Han, Y.-G. Kim, J. Y. Kim, and G. M. Lee, Biotechnol. Bioeng., 2010, 105, 1187. 
[48] K. Teter and R. K. Holmes, Infect. Immun. , 2002, 70, 6172.

[49] E. Baek, C. L. Kim, M. G. Kim, J. S. Lee, and G. M. Lee, Biotechnol. Bioeng., 2016, 113, 1953.

[50] H. Yoshida, K. Haze, H. Yanagi, T. Yura, and K. Mori, J. Biol. Chem., 1998.

[51] K. Kokame, H. Kato, and T. Miyata, J. Biol. Chem., 2001.

[52] S. H. Back, J. R. Hassler, and R. J. Kaufman, Protein Misfolding Disorders: A Trip into the ER. 2012.

[53] C. Jousse, C. Deval, A. C. Maurin, L. Parry, Y. Chérasse, C. Chaveroux, R. Lefloch, P. Lenormand, A. Bruhat, and P. Fafournoux, J. Biol. Chem., 2007, 282, 15851.

[54] S. Behrman, D. Acosta-Alvear, and P. Walter, J. Cell Biol., 2011, 192, 919.

[55] S. Saini, S. Yamamura, S. Majid, V. Shahryari, H. Hirata, Y. Tanaka, and R. Dahiya, Cancer Res., 2011, 71, 6208.

[56] M. H. Sahani, E. Itakura, and N. Mizushima, Autophagy, 2014, 10, 431.

[57] W. B’Chir, A. C. Maurin, V. Carraro, J. Averous, C. Jousse, Y. Muranishi, L. Parry, G. Stepien, P. Fafournoux, and A. Bruhat, Nucleic Acids Res., 2013, 41, 7683.

[58] M. Kaneko, S. Yasui, Y. Niinuma, K. Arai, T. Omura, Y. Okuma, and Y. Nomura, FEBS Lett., 2007, 581, 5355.

[59] Y. Ma and L. M. Hendershot, J. Biol. Chem., 2004, 279, 13792.

[60] M. A. Jardon, B. Sattha, K. Braasch, A. O. Leung, H. C. F. Côté, M. Butler, S. M. Gorski, and J. M. Piret, Biotechnol. Bioeng., 2012, 109, 1228.

[61] T. K. Ha, Y. G. Kim, and G. M. Lee, Biotechnol. Bioeng., 2015, 112, 1583.

[62] S. Tsuyuki, M. Takabayashi, M. Kawazu, K. Kudo, A. Watanabe, Y. Nagata, Y. Kusama, and K. Yoshida, Autophagy, 2014, 10, 497.

[63] A. Rojas-Fernandez, L. Herhaus, T. Macartney, C. Lachaud, R. T. Hay, and G. P. Sapkota, Sci. Rep., 2015, 5, 9811. 


\section{Figure legend}

Figure 1. [Characterisation of the recombinant CHO-EPO cell line and mRNA abundance. (A) Cell growth (in blue) and EPO volumetric productivity (in red) of CHO-EPO cell line. (B) Determination of phases of cell growth. Colours represent the different phases of growth ( exponen $\square \mathrm{l}$, stationar $\square$ and decline). $\square$ alues represent the average of three biological replicates \pm S.D. (C) Relative mRNA abundance of UPR-related targets and (D) housekeeping genes at day 3 of batch culture (exponential). Each count of the nCounter represents one mRNA transcript detected in $100 \mathrm{ng}$ of total RNA from the CHO-EPO cells. NanoString nCounter values are presented as the average of 2 replicate cultures \pm S.D.]

Figure 2. [UPR-related gene expression of CHO-EPO cell treated with $6 \mu \mathrm{g} / \mathrm{ml}$ tunicamycin for $0.5 \mathrm{~h}, 4 \mathrm{~h}$ and $24 \mathrm{~h}$. The means \pm S.D. are shown as the relative fold-induction when the values obtained in samples without tunicamycin were set as 1 . All samples were normalised against the four housekeeping genes using nSolver software. Unpaired student's t-test has been performed to compare mRNA expression between treated and untreated samples. The asterisk indicates a p-value of $\leq 0.05$ relative to the control]

Figure 3. [Profile of UPR-related mRNA expression during batch culture. Relative mRNA expression of UPR-related genes during batch culture in serum-free media was assessed by NanoString nCounter technologies. Day 1 mRNA expression was set as 1.0 for all the genes. All samples were normalised against the four housekeeping genes using nSolver software. Colours represent the different phases of growth ( exponentia $\square$ stationary a $\square$ decline). The $\square$ rror bars represent the standard deviations calculated from the data obtained from duplicate cell culture.]

Figure 4. [Transcriptional regulation of the unfolded protein response in mammalian cells. Stressed cells sense the accumulation of misfolded proteins in the ER lumen through three ER membrane-bound sensors (IRE1, PERK, and ATF6), which upon activation generate three transcription factors (XBP1s, ATF4 and ATF6(N) that transduce the signal to the nucleus to activate a transcriptional response mediated by ER stress responsive cis-acting elements present in the promoters of UPR-responsive genes. UPR-responsive genes present one or more ER stress-responsive cis-acting elements (ERSEI, ERSEII, UPRE or C/EBPATF/AARE) to which they can bind individually (i.e ATF4), as homodimers (i.e. XBP1sXBP1s) or as heterodimers with other transcription factors (i.e. ATF6(N)-NF-Y). These cisacting elements provide the differential sensitivity observed upon activation of the UPR arms. Despite that more than one UPR-associated transcription factor can bind to the same response element, the affinity between the transcription factors and the cis-acting element varies, which allows the generation of different cellular responses depending on the intensity with which each branch of the UPR is stimulated. ] 

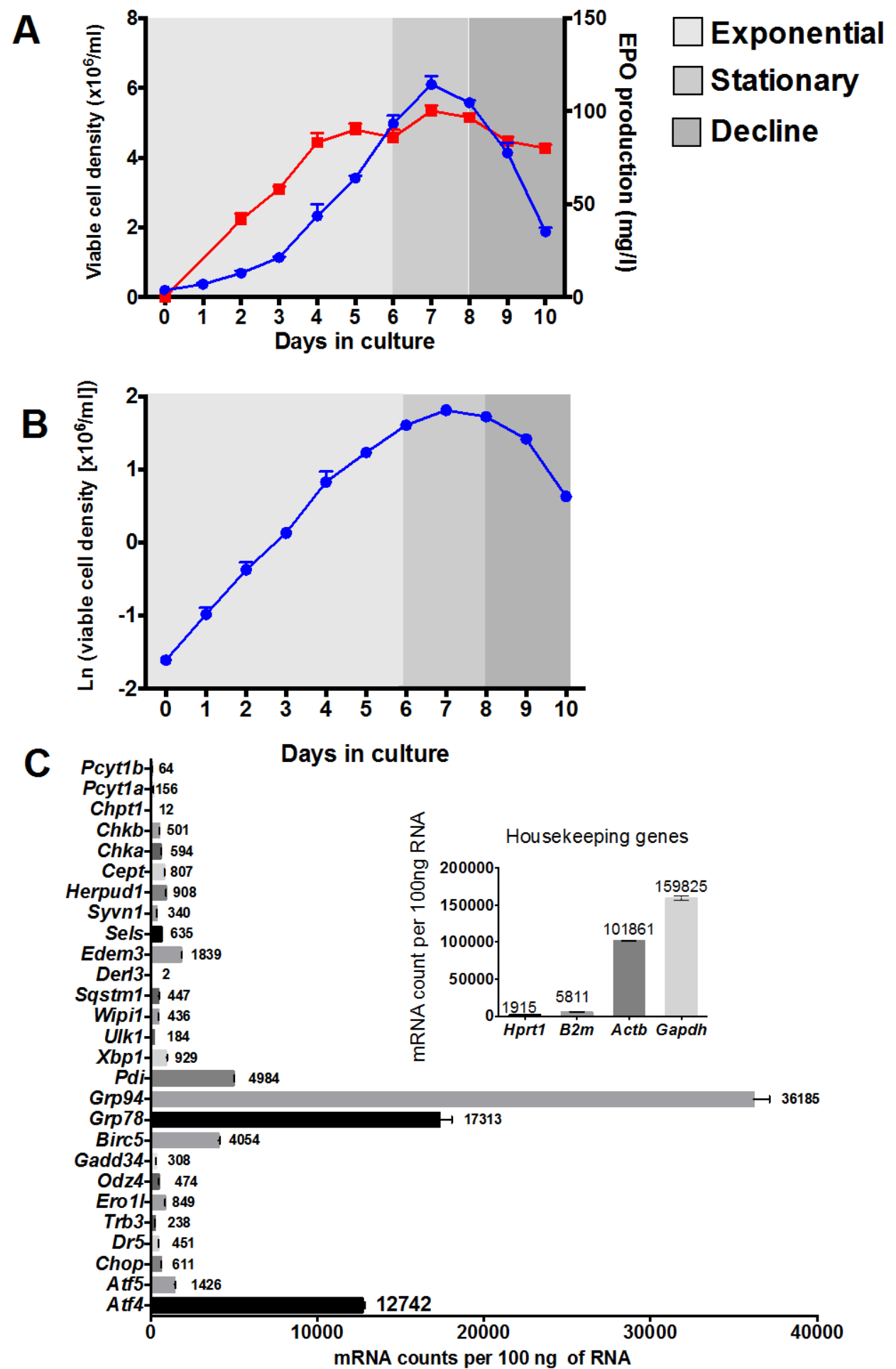

Figure 1 

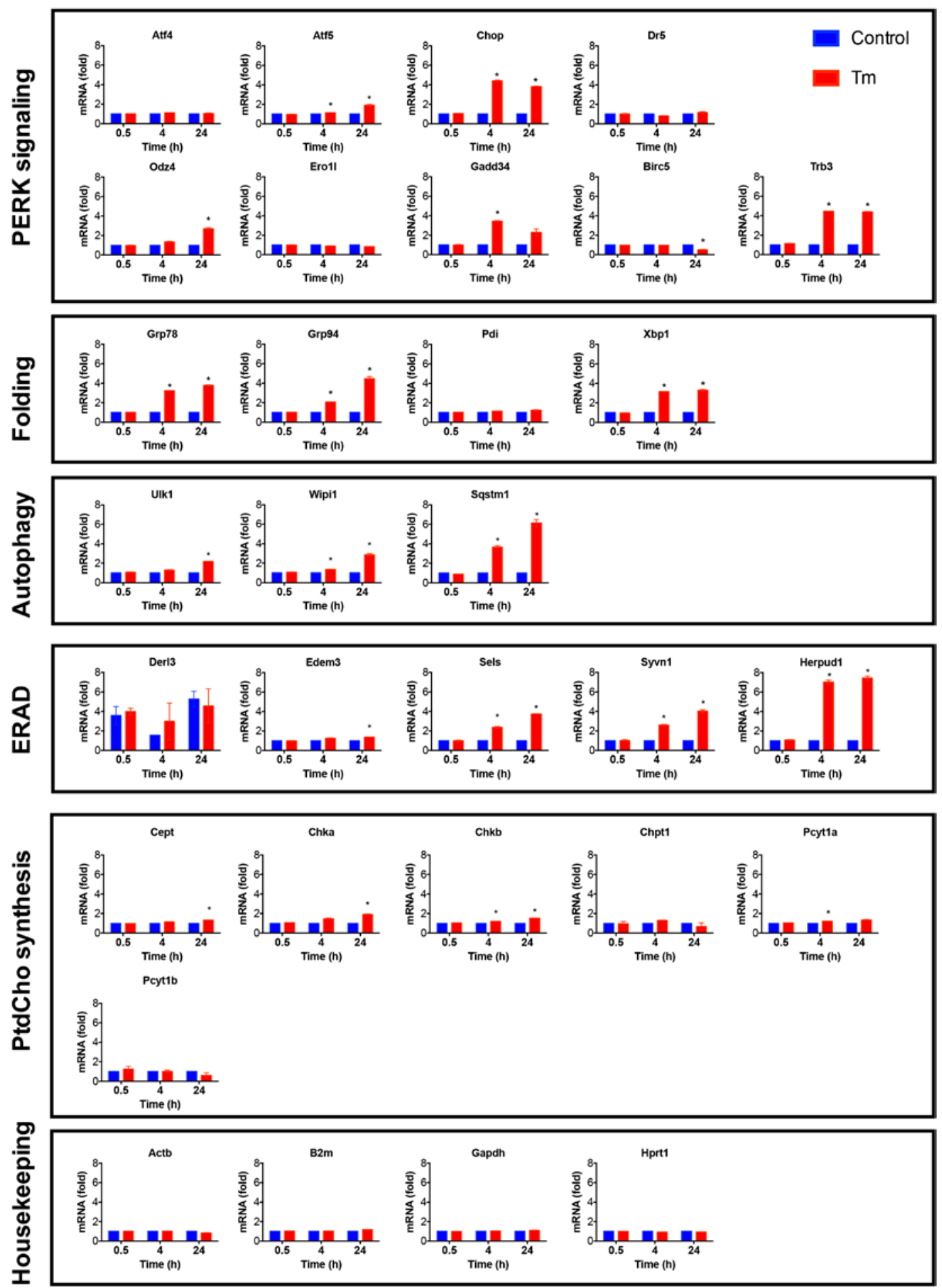

Figure 2 

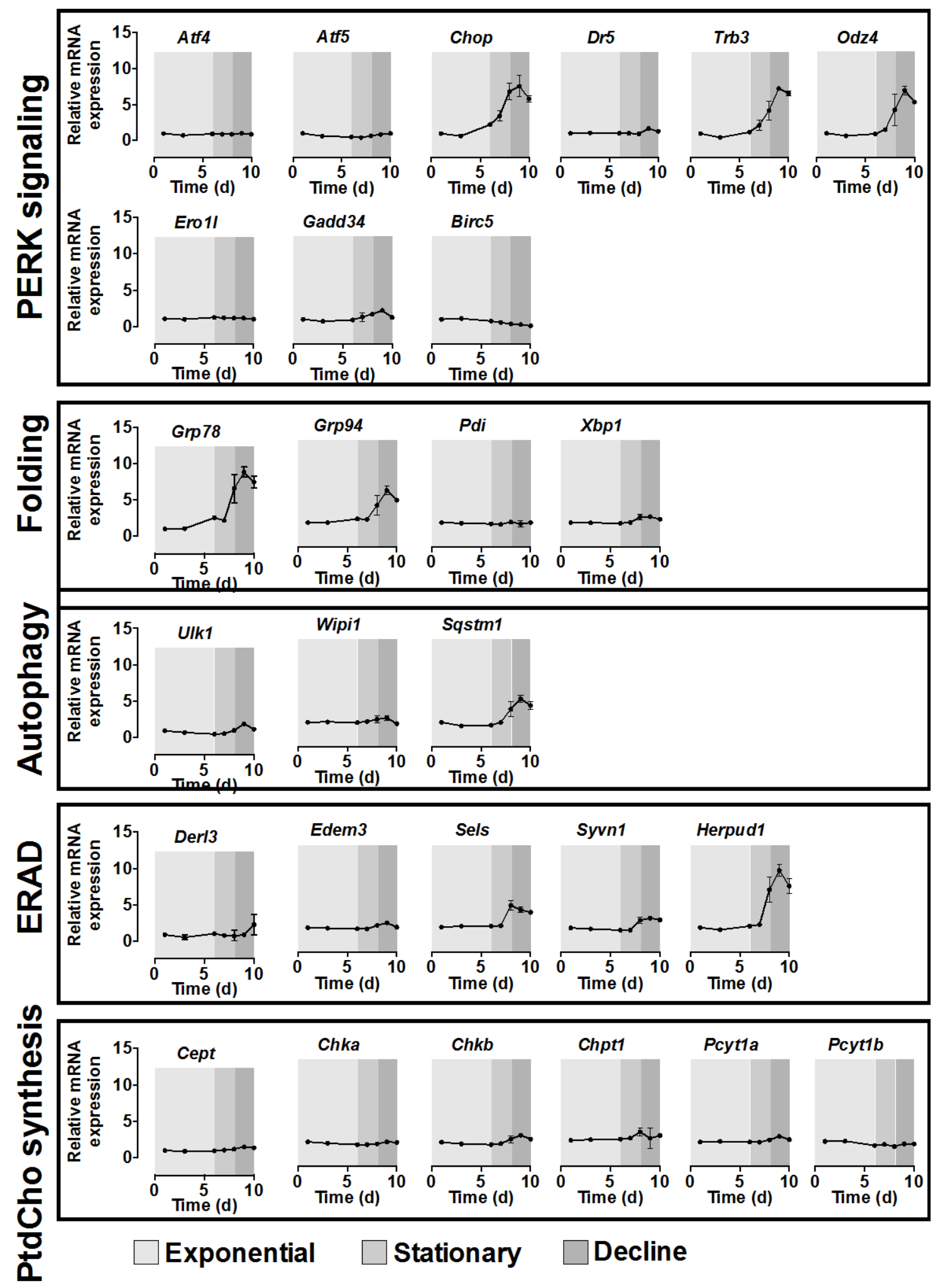

Figure 3 


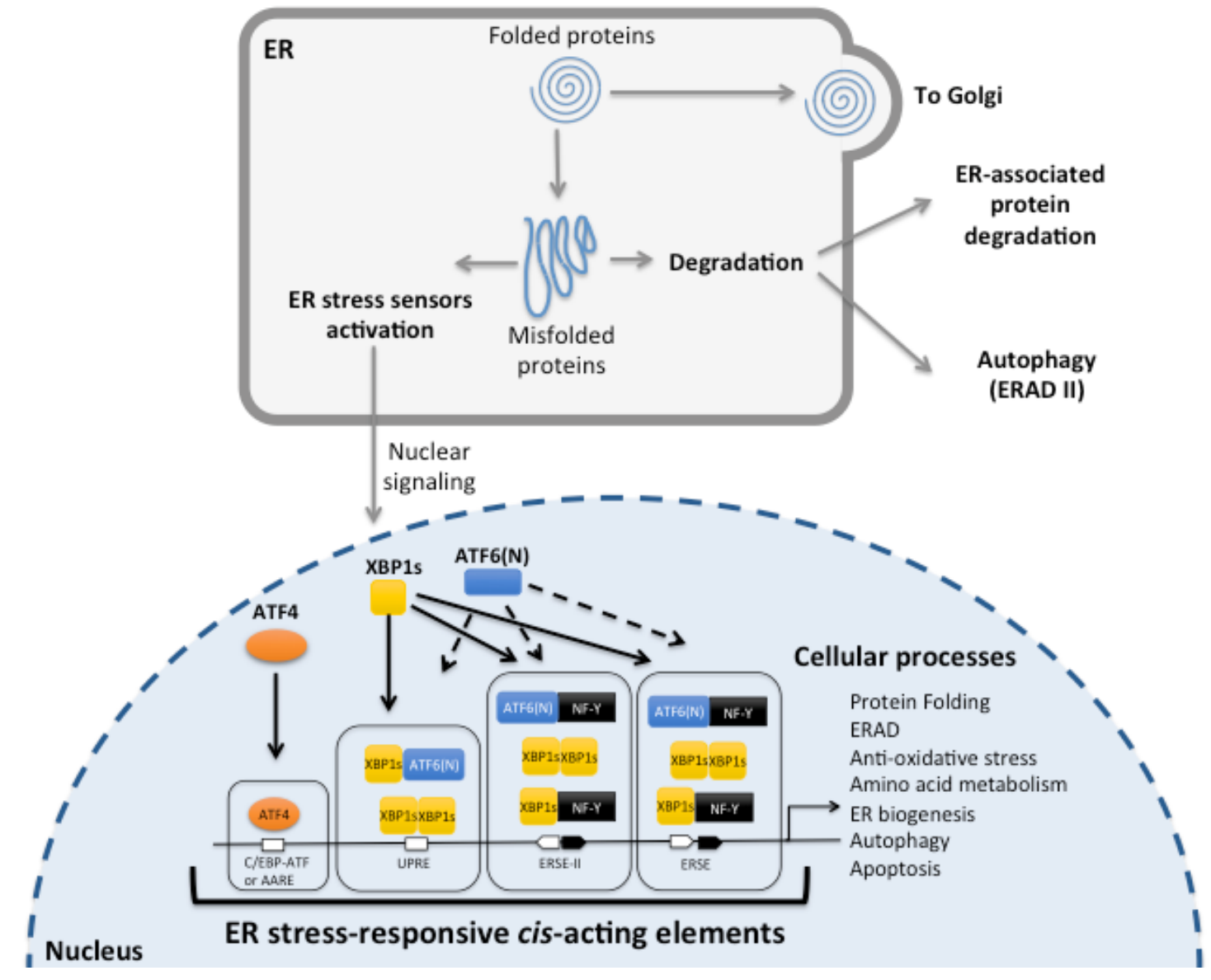

Figure 4 


\begin{tabular}{|c|c|c|c|}
\hline Group & Symbol & Accession No. & Gene \\
\hline \multirow{10}{*}{ PERK signalling pathway } & ATF4 & NM_001246812.1 & Activating transcription factor 4 \\
\hline & ATF5 & XM_003510884.2 & Activating transcription factor 5 \\
\hline & CHOP/GADD153/DDIT3 & XM_007648093.1 & C/EBP-homologous protein \\
\hline & DR5 & XM_003496176.2 & Death receptor 5 \\
\hline & TRB-3 & XM_003511497.2 & tribbles pseudokinase 3 \\
\hline & Odz4 & XM_003510673.1 & Odd Oz/ten-m homolog 4 \\
\hline & Ero1L & XM_007651327.1 & Endoplasmic reticulum oxidoreductin-1-like protein \\
\hline & Ppp1r15a/GADD34 & XM_007649270.1 & Protein phosphatase 1 , regulatory subunit $15 \mathrm{~A}$ \\
\hline & Birc5/Survivin & XM_007633873.1 & Baculoviral IAP repeat containing 5 \\
\hline & GRP78/BIP/HSPA5 & NM_001246739.2 & $78 \mathrm{kDa}$ glucose-regulated protein \\
\hline \multirow[t]{6}{*}{ Folding } & GRP94 & XM_003505850.2 & $94 \mathrm{kDa}$ glucose-regulated protein \\
\hline & PDI & NM_001246693.1 & Protein disulfide isomerase \\
\hline & XBP1 & NM_001244049.1 & X-box binding protein 1 \\
\hline & Cept & XM_003512375.2 & Choline/ethanolamine phosphotransferase 1 \\
\hline & Chka & XM_007654922.1 & Choline kinase alpha \\
\hline & Chkb & XM_003515462.2 & Choline kinase beta \\
\hline \multirow[t]{4}{*}{ Phosphatidylcholine synthesis } & Chpt1 & XM_003499706.2 & Choline phosphotransferase 1 \\
\hline & Pcyt1a & NM_001246737.1 & Phosphate cytidylyltransferase 1 , choline, alpha \\
\hline & Pcyt1b & XM_003505692.2 & Phosphate cytidylyltransferase 1 , choline, beta \\
\hline & ULK1 & XM_007642384.1 & Unc-51 like autophagy activating kinase 1 \\
\hline \multirow[t]{4}{*}{ Autophagy-related genes } & Wipi1 & XM_003500319.2 & WD repeat domain, phosphoinositide interacting 1 \\
\hline & Sqstm1 & XM_007645900.1 & Sequestosome 1 \\
\hline & Derl3 & XM_003508106.2 & Derlin 3 \\
\hline & EDEM-3 & XM_007641575.1 & ER degradation enhancer, mannosidase alpha-like 3 \\
\hline \multirow{6}{*}{$\begin{array}{l}\text { ER-associated degradation } \\
\text { (ERAD) }\end{array}$} & & & \\
\hline & Sels/VIMP & NM_001256848.1 & Selenoprotein S/VCP-interacting membrane protein \\
\hline & Syvn1/HRD1 & XM_003514586.2 & Synovial apoptosis inhibitor 1 \\
\hline & HERPUD1 & XM_003503612.2 & $\begin{array}{l}\text { Homocysteine-inducible, endoplasmic reticulum stress- } \\
\text { inducible, ubiquitin-like domain member } 1\end{array}$ \\
\hline & Actb & NM_001244575.1 & Actin beta \\
\hline & $\mathrm{B} 2 \mathrm{M}$ & NM_001246674.1 & Beta-2 microglobulin \\
\hline \multirow[t]{2}{*}{ Housekeeping } & GAPDH & NM_001244854.1 & Glyceraldehyde-3-phosphate dehydrogenase \\
\hline & HPRT1 & XM_003503017.1 & Hypoxanthine-guanine phosphoribosyltransferase 1 \\
\hline
\end{tabular}

Table 1: List of UPR-related selected genes 